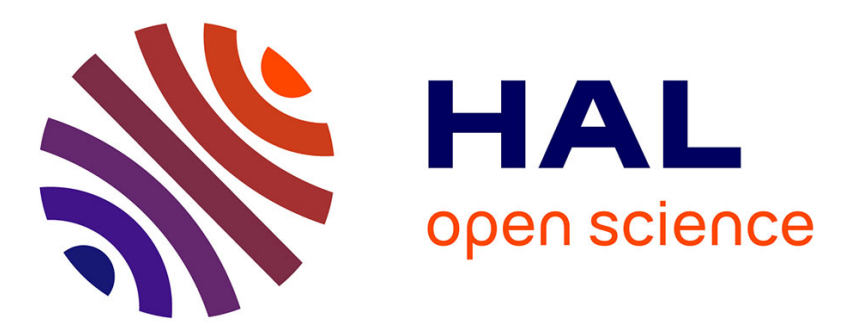

\title{
Structural studies of biologically active glycosylated polyamidoamine (PAMAM) dendrimers
}

Teresa Silva Barata, Sunil Shaunak, Ian Teo, Mire Zloh, Steve Brocchini

\section{To cite this version:}

Teresa Silva Barata, Sunil Shaunak, Ian Teo, Mire Zloh, Steve Brocchini. Structural studies of biologically active glycosylated polyamidoamine (PAMAM) dendrimers. Journal of Molecular Modeling, 2010, 17 (8), pp.2051-2060. 10.1007/s00894-010-0907-1 . hal-00652151

\section{HAL Id: hal-00652151 https://hal.science/hal-00652151}

Submitted on 15 Dec 2011

HAL is a multi-disciplinary open access archive for the deposit and dissemination of scientific research documents, whether they are published or not. The documents may come from teaching and research institutions in France or abroad, or from public or private research centers.
L'archive ouverte pluridisciplinaire HAL, est destinée au dépôt et à la diffusion de documents scientifiques de niveau recherche, publiés ou non, émanant des établissements d'enseignement et de recherche français ou étrangers, des laboratoires publics ou privés. 


\title{
Structural studies of biologically active glycosylated PAMAM dendrimers
}

Received: 15.09.2010 / Accepted: 05.11.2010

Teresa S. Barata ${ }^{1}$, Sunil Shaunak ${ }^{2}$, Ian Teo ${ }^{2}$, Mire Zloh $^{\bowtie}$, and Steve Brocchini ${ }^{1, \bowtie}$

${ }^{1}$ School of Pharmacy, University of London, 29 - 39 Brunswick Square, London WC1N 1AX, UK

${ }^{2}$ Department of Medicine, Imperial College London, Hammersmith Hospital, Ducane Road, London, W12 ONN, UK

${ }^{\square}$ Prof. Steve Brocchini: Email: steve.brocchini@pharmacy.ac.uk; Tel.: +44 207753 502; Fax.: +44 2077535964

${ }^{\otimes}$ Dr. Mire Zloh: Email: mire.zloh@ pharmacy.ac.uk, Tel.: +44 207753 5879, Fax.: + 4420 77535942

\begin{abstract}
The partial modification of carboxylic acid terminated polyamidoamine (PAMAM) dendrimers with glucosamine has been reported to give dendrimer glucosamine conjugates novel immuno-modulatory and anti-angiogenic properties. Experimental analysis of these glycosylated dendrimers showed that, on average, eight glucosamine molecules were covalently bound to each dendrimer. In order to better understand the surface loading and distribution of these glucosamine molecules, molecular reactivity was determined by evaluation of electronic properties using frontier molecular orbital theory (FMOT) and molecular dynamics simulations. It was shown that the surface loading and distribution of the zero length amide bond conjugated glucosamine molecules was determined by both electronic effects and by the different dynamic conformations adopted by the modified dendrimer during the incremental addition of glucosamine. Importantly, the structural features and the dynamic behavior of the partially glycosylated generation 3.5 PAMAM dendrimer showed that its flexibility and its polarity changed with the incremental addition of glucosamine. These
\end{abstract}


peripheral glucosamine molecules remained available on the dendrimer's surface for interaction with the biological target.

Keywords PAMAM dendrimers $\cdot$ Anti-inflammatory $\cdot$ FMOT $\cdot$ Molecular dynamic simulations 


\section{Introduction}

The partial modification of carboxylic acid terminated polyamidoamine (PAMAM) dendrimers with glucosamine has been reported to give dendrimer glucosamine conjugates novel immuno-modulatory and anti-angiogenic properties [1]. Dendrimers are a class of polymeric compounds that can be distinguished from conventional linear polymers by their highly branched and symmetrical architecture [2]. Dendrimers are synthesized using a sequence of reactions, each time the sequence is conducted, the dendrimer is said to increase by one generation. The number of end groups double and the molecular weight of the dendrimer increases each time the sequence of reactions is conducted. The generation of a dendrimer is representative of both its size (i.e., diameter in angstroms) and its molecular weight [3].Typically, PAMAM dendrimers are available in whole number generations (amine terminated). If the surface end groups are capped, the dendrimer is said to have increased by a half-generation. Hence for an amine terminated dendrimer of generation 3, capping it to have carboxylic acid terminated end groups will give a generation 3.5 dendrimer. The number of end groups does not change by simple capping of the existing endgroups. The PAMAM dendrimer used was a generation 3.5 with 64 carboxylic acid end groups. These were prepared by carbodiimide based coupling to form a stable zero length amide bond between the carboxylic acid terminated dendrimer and the aminosaccharide (Scheme 1). The divergent synthesis used does not allow for predetermined positioning of the glucosamine molecules on the dendrimer's surface.

\section{$\langle$ Scheme 1>}

Experimental analysis of the glycosylated dendrimers showed that, on average, eight glucosamine molecules were covalently bound to each dendrimer. In order to better understand the surface distribution of these glucosamine molecules, and its contribution to the biological properties of the dendrimer glucosamine molecule, we have developed a method to generate 3D models of these dendrimers in silico [4]. In this paper, these structures have been used to computationally study the loading and the distribution of glucosamine on the end groups of the PAMAM dendrimer, and to define the dynamic behavior of the whole molecule.

Molecular reactivity can be estimated by evaluation of electronic properties. The frontier molecular orbital theory (FMOT) focuses on valence electrons and the interaction between the highest occupied molecular orbital (HOMO) and the lowest unoccupied molecular orbital 
(LUMO) in the molecules involved in both the formation and the breaking of bonds [5]. Chemical reactions are favored at atoms in molecules where the maximum overlap between the HOMO of a reactant molecule and the LUMO of another reactant molecule leads to bond formation [6]. The FMOT has been widely used for the study of chemical reactions; e.g., Diels Alder reactions [7] and metal substitutions [8]. For dendrimeric structures, this theory has been used to study energy flow and the intensity of light emission from a nanostar dendrimer[9].

Although the electronic effects can have a determining effect on reactivity, molecular conformational and dynamic behaviors can also play important roles when macromolecules such as dendrimers are considered. Molecular dynamics studies of PAMAM dendrimers have already helped to elucidate the structural features and behavior of these molecules. A study varying the percentage acylation of a generation 5 PAMAM dendrimer showed that their overall shape was not dependent upon the percentage of acyl groups on their surface because intramolecular hydrogen bonds form and break during the simulation [10].

In this paper, frontier molecular orbital theory coupled with molecular dynamics simulations were used to understand the structural features of the experimentally determined loading and distribution of covalently bound glucosamine, and its impact on the surface properties of the PAMAM dendrimer.

\section{Experimental section}

\section{Molecular dynamics simulation of saccharide modified dendrimers}

The dendrimer structures were generated with X-PLOR using a recently described method and saved in pdb format [4]. VMD [11] was used to convert the structures into the mol2 file format and then imported into Maestro [12]. The dendrimer system was prepared for molecular dynamics simulations using Desmond with explicit solvent [13]. The dendrimer glucosamine system was built using the SPC solvation model and the size of the box was determined automatically by creating a $10 \AA$ buffer zone around the dendrimer. The molecular dynamics simulation, structure minimization and relaxation steps were performed for $4.8 \mathrm{~ns}$ at $300 \mathrm{~K}$ and 1.03 bar. Snapshot structures were recorded every 4.8 ps. 


\section{Trajectory analysis of the molecular dynamics simulation}

The trajectory from Desmond output in ".cms" format was loaded into VMD where water and ions were removed and a new trajectory was exported as a mol 2 file. These files were loaded in VegaZZ 2.2.0.54 [14] and the trajectory analysis tool used to determine gyration radius, polar surface area (PSA), surface area and RMSD. The resulting values were imported into Microsoft Excel and plotted. Both the distance from the core to the surface glucosamine and the number of hydrogen bonds were determined using the Maestro measuring toolkit. Accelerys Discovery Studio 2.5 (http://accelrys.com/products/discovery-studio/) was used to generate the interpolated charge surfaces.

\section{Determination of electronic properties by semi-empirical methods}

The electronic properties of the dendrimers were determined by semi-empirical methods using Mopac 2009 9.303W. Input files for Mopac were created using VegaZZ 2.2.0.54 interface, choosing PM6 and the key words 1SFC, MMOK, GRAPHF and VECTORS. Due to computational restrictions, the molecules submitted for these calculations were a one quarter 'slice' of the representative conformations of the generation 3.5 PAMAM dendrimer and dendrimer glucosamine conjugate as generated by molecular dynamics simulations. These calculations were conducted on a quarter of the generation 3.5 PAMAM dendrimer:- (a) without any modifications (qdend); (b) modified with one glucosamine (qd1s); (c) modified with two glucosamine molecules (qd2s); and (d) modified with three glucosamine molecules (qd3s). In each case, six representative conformations of each dendrimer structure were subjected to calculations. They were used to approximate electronic properties that reflected the electronic properties of the whole PAMAM dendrimer with different loadings of glucosamine.

\section{Results and discussion}

The three dimensional structures of five generation 3.5 PAMAM dendrimers with different loadings of glucosamine were generated and the resulting structures submitted to molecular dynamics simulations with 1000 structures saved in each trajectory. These were then clustered to select a significant number of representative structures to study the loading and distribution 
of glucosamine. Additionally, the whole trajectory was analyzed for a better understanding of the dynamic behavior of these dendrimers with and without conjugated glucosamine.

\section{Loading and distribution of the glucosamine molecules conjugated to a generation $\mathbf{3 . 5}$ PAMAM dendrimer}

The generation 3.5 PAMAM dendrimer displays 64 carboxylic end groups that are, in theory, all available for covalent modification with glucosamine. NMR studies of the glucosamine PAMAM dendrimer conjugates have shown that there are, on average, eight glucosamine molecules per dendrimer [1] \& unpublished observations].

Frontier Molecular Orbital Theory (FMOT) postulates that the interaction between the HOMO and the LUMO of molecules undergoing a reaction can provide a good approximation of reactivity [5]. A reaction will occur with increasing and often maximal overlap of the HOMO and LUMO orbitals. For this study, the LUMO of the glucosamine molecule and the HOMO of the dendrimer with different patterns of glycosylation was determined. The difference in the energy between the dendrimer HOMO and glucosamine LUMO was then determined and analyzed.

The large size of the dendrimer (894 heavy atoms) meant that only partial segments of the structure could be readily used to determine the HOMO-LUMO electronic properties, and that semi-empirical methods would be applicable. The HOMO of the dendrimer was located on one of the terminal carboxylic acid groups. This would allow an interaction with the LUMO in the glucosamine, which was found to be located on the amine group (Fig. 1).

$<$ Figure 1>

These electronic property calculations were applied to increasing sizes of dendrimer branches with and without glucosamine (Fig. 2). This approach enabled confirmation that the position of the HOMO was in the terminal carboxylic groups where the interaction with the LUMO of glucosamine was expected to take place. An important new observation was that when the first glucosamine molecule was covalently attached to the dendrimer, the HOMO was always located on the opposite branch to the one with the zero length amide bond between the dendrimer and the glucosamine.

$<$ Figure 2> 
The gap between the HOMO of the dendrimer and the LUMO of the glucosamine decreased as the size of the dendrimer molecule increased. According to the FMOT, the smaller the gap between the HOMO and LUMO the more likely is the reaction to take place. By analogy, it was expected that this gap would be even smaller if the whole dendrimer was considered.

Computational limitations meant that a quarter of the dendrimer structure (i.e., a "slice" displaying 16 terminal carboxylic groups) was selected for further detailed analysis. Dendrimers are hyperbranched and flexible molecules with many rotatable bonds. Six representative conformations from a quarter section of the dendrimer without glucosamine, and the same number of conformations of the dendrimer with 1,2 and 3 glucosamine molecules were considered in relation to the LUMO of the glucosamine molecule $(-4.61 \mathrm{eV})$. The averaged energy values of the HOMO for each dendrimer can be found in the table below (Table 1).

$<$ Table 1>

It was found that the $\mathrm{HOMO}^{\mathrm{d}} / \mathrm{LUMO}^{\mathrm{s}}$ gap increased as additional glucosamine molecules were covalently bound to the dendrimer. According to FMOT, the increase in the gap indicated that each addition of glucosamine decreased the overlap of the HOMO and the LUMO. This thereby decreased the probability that additional conjugation reactions would occur with the adjacent surface carboxylic acid groups of the dendrimer.

A detailed analysis of the dendrimer segments showed that the energies of both the HOMO and HOMO-1, with three glucosamine molecules attached, was almost double the LUMO energy value of the glucosamine molecule. This would make the addition of a fourth sugar to the dendrimer section under study very unlikely. Therefore, the theoretical maximum loading of a generation 3.5 PAMAM dendrimer with glucosamine was found to be 12 .

However, electronic effects on their own, are insufficient to completely understand the loading and distribution of the glucosamine molecules on the surface of these dendrimers. Observation of the position of the HOMO, and defining both the location and availability of the terminal group, was necessary to better understand the loading and distribution of the glucosamine molecules (Fig. 3 and Table 2).

$<$ Figure 3> 
Both the HOMO and HOMO-1 positioning and availability were inspected for each structure. Fig. 3 shows an example of the HOMO position for each of the quarter segments of the dendrimers that were studied. The first structure is representative of the six conformations for the segment of the dendrimer with no glucosamine attached. The HOMO/HOMO-1 orbitals are located at the end groups of the branches of the dendrimer and they are always available for the conjugation reaction.

The second structure corresponds to one of the six conformations with one glucosamine conjugated to the surface of the dendrimer. It can also be considered representative of all six conformations in which the HOMO is always available. For these molecules, the HOMO, though not always located in the extremities or at the end groups of the branch, is always at a considerable distance from the conjugated glucosamine molecule. In this case, the HOMO-1 did not follow the trend described in that it was only available in half of the structures studied (Table 2). The third structure (Fig. 4) corresponds to one of the six conformations of the quarter segment of the dendrimer with two glucosamine molecules conjugated to its surface. This structure was also representative of what happens with these structures when the HOMO is located in the interior of the dendrimer's branches. This affects its availability for reaction with glucosamine. The last structure shown (Fig. 4D) corresponds to one of the six conformations of the quarter of the dendrimer with three glucosamine molecules conjugated to its surface. In this case, the HOMO is never available on the surface and it appears on an end group at the extremity of the branch. Furthermore, it points inwards towards the core of the dendrimer. As a result, the adjacent branch from the whole dendrimer sterically interferes with the end group. The availability of the HOMO and HOMO-1 for all the representative structures under study are summarized in Table 2.

$<$ Table 2>

The addition of the first glucosamine is favorable because the HOMOs of the carboxylic groups are available. After one saccharide has covalently bound to one of the end groups, the HOMO is again located on a peripheral carboxylic acid group making the addition of a second glucosamine favorable. When two sugars are bound, in a quarter segment of the dendrimer, it becomes much less likely that the addition of third glucosamine will take place because there is only a 50:50 chance that the HOMO will be available for interaction with a carboxylic acid end group. The addition of a fourth glucosamine molecule is very unlikely to occur within the same quarter segment of the dendrimer. 
Taking both electronic and steric effects into account, the conjugation of 4 to 8 glucosamine molecules onto the available 64 carboxylic acid end groups that are present on the generation 3.5 PAMAM dendrimer is the most favorable energetic and steric outcome. The conjugation of additional glucosamine molecules requires overcoming increasingly higher energy barriers with available positions for conjugation being available for only half of the time. Additional conjugation reactions of glucosamine beyond twelve molecules on the whole dendrimer are very unlikely because the energy gap and the overlap between the LUMO of glucosamine and the HOMO of the newly formed conjugate is large, and the carboxylic acid end group is not available. These molecular modeling based observations are consistent with the experimental data that an average of 8 glucosamine molecules were conjugated to the surface of a generation 3.5 PAMAM dendrimer.

\section{Molecular dynamics simulation analysis of glycosylated PAMAM dendrimers}

To better understand the dynamic behavior of these dendrimers, molecular dynamics simulations were carried out for $4.8 \mathrm{~ns}$. Molecular properties including the surface area, polar surface area, gyration radius and RMSD were estimated and plotted as a function of time using the VegaZZ trajectory analysis tool (Fig. 4).

\section{$<$ Figure 4>}

The surface area graph (Fig. 4B) shows that there is an initial decrease in the surface area values. This is most likely to be the result of the starting structure being generated in vacuum and the use of nOe constraints to stretch the initial structure. After an initial decrease, the area oscillated around the average value of $13965 \AA^{2}$. The polarity of the surface (Fig. 4C) followed a similar pattern along the trajectory.

The RMSD analysis, as a measure of flexibility and folding [15-17], was conducted for all of the PAMAM dendrimer structures. A plateau was seen after the first 1400 ps (Fig. 4D). This means that the molecule explored the conformational space with similar differences when compared to the initial structure set as reference for the RMSD calculations. However, further changes in the explored conformational space were observed as indicated by the increase of the RMSD values towards the end of the simulation. Although the RMSD reached a plateau after $1.4 \mathrm{~ns}$, RMSD values of approximately $11 \AA$ indicated a flexible structure. This plateau was consistent with the profile that was obtained for the gyration radius where, after only 
$1400 \mathrm{ps}$, limited fluctuations occurred. These fluctuations indicated that the overall shape of the structure was not undergoing major changes during the simulation period.

Closer inspection of the overlay of representative structures also indicated that the core and inner branching units had little mobility with most of the RMSD changes occurring because of movement of the outer sphere of the dendrimer (Fig. 5). The three superimposed structures, with overlapped dendrimer cores, at three different time points indicated that the first branching generation of the dendrimer did not change during the simulation. From the second generation onwards, and all the way to the end groups, the structures showed varying degrees of flexibility. The greatest flexibility was observed in the last 1.5 generations of the dendrimer. This was consistent with the observations described above.

$<$ Figure 5>

To examine the influence of the conjugation of glucosamine on the conformation and the molecular properties of these surface modified PAMAM dendrimers, molecular dynamics simulations were performed with three different loadings of glucosamine of the generation 3.5 PAMAM dendrimer. The entire dendrimer was modified with seven, eight or nine glucosamine molecules and their molecular properties determined and compared as shown in Fig. 6.

$<$ Figure 6>

These three glycosylated dendrimers displayed very similar gyration radius values. Throughout the simulation, the dendrimer with nine glucosamine molecules was the most compact. For the surface area values, the three glycosylated dendrimers displayed profiles with similar values with the nine glucosamine dendrimer molecule having lower values than the eight glucosamine molecule for a significant period of time. This was consistent with the nine glucosamine dendrimer having a more compact structure, an observation that was not expected because this molecule had the highest number of atoms.

The surface polarity of these glycosylated dendrimers was proportional to the number of conjugated glucosamines. Finally, for the RMSD values, the dendrimer with eight glucosamines attached was clearly different. This suggested that this molecule explored a larger conformational space because of its even distribution of surface glucosamine molecules. However with longer simulation times, these properties reached a plateu and 
converged. The observed difference in behavior of the dendrimer with eight glucosamines on its surface from the other two dendrimer glucosamine conjugates was interesting because it was the only molecule with a even distribution of surface glucosamine molecules. It is the most flexible of the three molecules. This could be an indication that the uneven distribution of glucosamine molecules leads to a rigid dendrimeric structure with smaller conformational space.

For all of these glycosylated dendrimers, their molecular properties converged and stabilised after 4 ns. Therefore, a single dendrimer glucosamine could be used as a representative structure for all of these molecules with different loadings of glucosamine. Similarly, molecular dynamics studies of heterogeneously modified PAMAM dendrimers showed that the distribution of their surface modifying groups was dependent upon the conformation of the core and its branching monomers, and that its overall shape was not altered by the surface modifications [18]. Therefore, based on the computational HOMO-LUMO reactivity studies and the analytical studies of dendrimer glucosamine conjugates, the generation 3.5 PAMAM dendrimer with eight glucosamine molecules conjugated via a zero length amide bond was found to be a good representative structure for further studies and analysis.

Others have also studied the effects of terminal group modifications on the solution properties of dendrimers using molecular dynamics. For example, the flexibility and shape of glycodendrimers [19] and poly(L-lysine) dendrimers[20] were studied. These properties were also studied using different monomers, generations, and different surface monomers [19, 20].

Our strategy was different in that we compared and contrasted biologically active (i.e., generation 3.5 PAMAM dendrimer modified with eight glucosamine molecules) and biologically inactive (i.e. generation 3.5 PAMAM dendrimer and a generation 3.5 PAMAM dendrimer modified with one glucosamine molecule) by analysing the flexibility and the shape of the molecule. Gyration radius, as a measure of molecular shape indicated that the presence of eight glucosamine molecules led to a less compact structure (Fig. 7A).

$<$ Figure 7>

The RMSD values are a measure of flexibility in that any value greater than $4 \AA$ is considered to be a different conformation. The dendrimer that has no glucosamine molecules conjugated to its end groups reaches a plateau rapidly during the 2 ns simulation (Fig. 7B). The units on 
the outer surface continued to change their conformation up to the end of the trajectory when it started to explore a different conformational space. The dendrimer with eight surface glucosamines presented several plateaus in its profile (before $2 \mathrm{~ns}$, at $2.5 \mathrm{~ns}$, and after $4 \mathrm{~ns}$ ). This means that it passed through several significantly different conformations. With time, this dendrimer glucosamine explored an increasingly larger conformational space (Fig. 7B).

Considering the profiles of these biologically active and inactive molecules led to the conclusion that the dendrimer with eight surface glucosamine molecules was the most flexible molecule because it explored the largest conformational space. This indicated that flexibility was an important property for the biological properties of these molecules.

We have also studied the number of intermolecular hydrogen bonds that the dendrimer with eight glucosamine molecules can form with the interior of the dendrimer. In the whole trajectory, it was found that: (a) in $26 \%$ of the conformations, one hydrogen bond was observed within the branching units; (b) in $8 \%$ of the conformations, two hydrogen bonds were observed within the branching units; (c) in $1.7 \%$ of the conformations, three hydrogen bonds were observed within the branching units; and (d) in only one structure were four hydrogen bonds observed within the branching units (Fig. 8). This raised the possibility that the branches bearing glucosamine molecules could be folding inwards towards the center of the dendrimer. Such inward folding would lead to a diminished capacity of the glucosamine moieties to be available for intermolecular interactions. Visual inspection of the structures was therefore performed and the glucosamine molecules found to be mostly outward facing and accessible (Fig. 8).

$<$ Figure 8>

Charge and its surface distribution were also studied. Interpolated charge surfaces were generated for the biologically inactive generation 3.5 PAMAM dendrimer and for the biologically active generation 3.5 PAMAM dendrimer with eight surface glucosamine molecules. The surfaces studied revealed a positively charged region throughout the branching dendrimer, and at the core of the dendrimer, where the protonated amine groups were located (Fig. 9). The negatively charged areas were found on the surface where the deprotonated carboxylic groups were present. The main differences between the PAMAM dendrimer and the glycosylated PAMAM dendrimer were seen on the surface of the molecule. The presence of the eight glucosamine molecules reduced the negative charge on the surface 
(Fig. 9B). This allowed for a more relaxed structure, a higher surface area, and a more balanced overall charge distribution. This could be making an important contribution to the biological activity of the molecule.

$<$ Figure 9>

\section{Conclusions}

These electronic studies describe the relative roles, contribution and importance of both the peripheral carboxylic acid end groups and of the surface loading and distribution of glucosamine molecules on generation 3.5 PAMAM dendrimers to their observed biological activity. The structural heterogeneity of the molecule was a consequence of both electronic effects and of the different conformations that the molecule could adopt. Taken together, these studies showed that the dynamic flexibility of the generation 3.5 PAMAM dendrimer, modified with an average of eight evenly distributed surface glucosamine molecules, was an important property of this biologically active molecule. Additional factors identified as being important were the conformational space explored by the dendrimer glucosamine, its overall surface area, and its surface polarity. The availability of peripheral glucosamine molecules for interaction with the biological target was not impaired by intramolecular hydrogen bonds.

\section{Acknowledgments}

We are grateful for funding from the US National Institutes of Health (NIH) (U01AI07535102). 


\section{References}

1. Shaunak S et al (2004) Nat Biotechnol 22:977-984

2. Vögtle F, Richardt G, Werner N (2009) Dendrimer Chemistry. Wiley-VCH Verlag GmbH \& Co KGaA, pp 1-24

3. Menjoge AR, Kannan RM, Tomalia DA (2010) Drug Discovery Today 15:171-185

4. Barata TS et al (2010) J Mol Model submitted

5. Fukui K (1982) Science 218:747-754

6. Fukui K (1971) Acc Chem Res 4:7-64

7. Wang $\mathrm{H}$ et al (2005) J Org Chem 70:4910-4917

8. Chen P, Solomon EI (2002) J Inorg Biochem 88:368-374

9. Tada T et al (2003) J Phys Chem B 107:14204-14210

10. Lee H, Baker JR, Larson RG (2006) J Phys Chem 110:4014-4019

11. Humphrey W, Dalke A, Schulten K (1996) J Mol Graph 14:33-38

12. Schrödinger Inc [cited; Available from: www.schrodinger.com

13. Levy RM, Gallicchio E (2003) Annu Rev Phys Chem 49:531-567

14. Pedretti A, Villa L, Vistoli G (2004) J Comput Aided Mol Des 18:167-173

15. Maiorov VN, Crippen GM (1994) J Mol Biol 235:625-634

16. Damm KL, Carlson HA (2006) Biophys J 90:4558-4573

17. Evangelos AC, Chaok S, Ken AD (2004) J Comput Chem 25:1849-1857

18. Roberts BP et al (2009) Macromolecules 42:2784-2794

19. von der Lieth CW, Frank M, Lindhorst TK (2002) Rev Mol Biotech 90:311-337

20. Roberts BP et al (2009) Macromolecules 42:2775-2783 


\section{Tables}

Table 1 Average values of the HOMO energy determined for each of the six representative conformations of each dendrimer studied, and differences between the HOMO of the quarter segment of the generation 3.5 PAMAM dendrimer and the LUMO of the glucosamine (-4.61 eV). $\mathrm{HOMO}^{\mathrm{d}}$-dendrimer orbital; $\mathrm{LUMO}^{\mathrm{s}}$ - glucosamine orbital

\begin{tabular}{ccccc} 
Molecule & $\begin{array}{c}\text { Number of } \\
\text { glucosamines }\end{array}$ & $\begin{array}{c}\text { HOMO }^{\mathbf{d}} \mathbf{- 1} \\
(\mathbf{e V})\end{array}$ & $\begin{array}{c}\text { HOMO }^{\mathbf{d}} \\
(\mathbf{e V})\end{array}$ & $\begin{array}{c}\text { HOMO }^{\mathrm{d}} \text {-LUMO } \\
(\mathbf{e V})\end{array}$ \\
\hline Qdend & 0 & -6.13 & -5.97 & 1.36 \\
qd1s & 1 & -7.46 & -7.32 & 2.71 \\
qd2s & 2 & -8.03 & -7.71 & 3.10 \\
qd3s & 3 & -9.00 & -8.80 & 4.19
\end{tabular}


Table 2 Availability of the terminal carboxylic groups where the HOMO and HOMO - 1 are located for each conformation and molecule studied. + available; - not available for the reaction

Number of sugars on the quarter of the gen. 3.5 PAMAM dendrimer
Availability of the HOMO

Availability of the HOMO-1

\begin{tabular}{ccc} 
dendrimer & \\
\hline 0 & +-++++ & ++++++ \\
1 & +++++- & +-++- \\
2 & ++--+ & ++-++ \\
3 & +---- & ---
\end{tabular}




\section{Figure captions}

Scheme 1 Synthesis of dendrimer glucosamine

Fig. 1 The HOMO orbital from a PAMAM dendrimer portion of a carboxylic acid end group (left) and the LUMO orbital of a glucosamine molecule (right). These electronic properties were calculated using PM6 method implemented in MOPAC

Fig. 2 Top - Increasing branches of PAMAM dendrimer modified with one glucosamine submitted to semiempirical calculations with Mopac to determine the HOMO energy value and its location (arrow). Bottom - HOMO ${ }^{\mathrm{d}}-\mathrm{LUMO}^{\mathrm{s}}$ gap as defined by the $\mathrm{HOMO}^{\mathrm{d}}$ energy value determined for each dendrimer structure and the LUMO of a glucosamine molecule $(-4.61 \mathrm{eV})$

Fig. 3 Position of the HOMO orbital (arrow) on a quarter segment of a generation 3.5 PAMAM dendrimer with increasing numbers of glucosamine molecules (shaded circle). (A) No glucosamine; (B) One glucosamine; (C) Two glucosamines; (D) Three glucosamines

Fig. 4 Molecular properties determined for a generation 3.5 PAMAM dendrimer along a trajectory of a $4.8 \mathrm{~ns}$ molecular dynamics simulation. (A) Gyration radius in Angstroms; (B) Surface area in square Angstroms; (C) Polar surface area in square Angstroms; (D) Root mean square (RMSD) in Angstroms

Fig. 5 Overlap of a quarter segment of a generation 3.5 PAMAM dendrimer at different time points of the trajectory of a $4.8 \mathrm{~ns}$ molecular dynamics simulation. Blue Structure after $1.4 \mathrm{~ns}$ of the simulation; Dark pink - Structure after $3 \mathrm{~ns}$ of the simulation; Red - Structure after 4.2 ns of the simulation. Black - Dendrimer core, superimposition of the core of the dendrimer

Fig. 6 Molecular properties determined for generation 3.5 PAMAM dendrimer modified with 7,8 or 9 glucosamine molecules along a trajectory of a $4.8 \mathrm{~ns}$ molecular dynamics simulation. Brown - dendrimer modified with seven glucosamine molecules; Green - dendrimer modified with eight glucosamine molecules; Blue - 
dendrimer modified with nine glucosamine molecules; (A) Gyration radius in Angstroms; (B) Surface area in square Angstroms; (C) Polar surface area in square Angstroms; (D) Root mean square deviation (RMSD) in Angstroms

Fig. 7A Gyration radius values determined for generation 3.5 PAMAM dendrimer modified with eight glucosamine molecules along a trajectory of a $4.8 \mathrm{~ns}$ molecular dynamics simulation. Blue - dendrimer without glucosamine molecules; Green - dendrimer modified with eight glucosamine molecules

Fig. 7B RMSD values determined for generation 3.5 PAMAM dendrimer modified with one or eight glucosamine molecules along a trajectory of a $4.8 \mathrm{~ns}$ molecular dynamics simulation. Blue - dendrimer without glucosamine molecules; Green dendrimer modified with eight glucosamine molecules

Fig. 8 Structure of a glycosylated PAMAM dendrimer that displayed a highest number of intramolecular hydrogen bonds between the glucosamine molecules and the dendrimer's branching units. The branching units are represented as the molecular surface. The terminal units are represented as tube and the glucosamine molecules as CPK. Only polar hydrogen atoms are displayed. The green arrow points to the only glucosamine that is buried on the molecular surface

Fig. 9 Interpolated charge surface for: (A) a generation 3.5 PAMAM dendrimer; (B) a generation 3.5 PAMAM dendrimer with eight surface glucosamine molecules. The red arrows point out three of these glucosamine molecules. The shaded red areas correspond to negatively charged residues and the blue areas to positively charged residues 


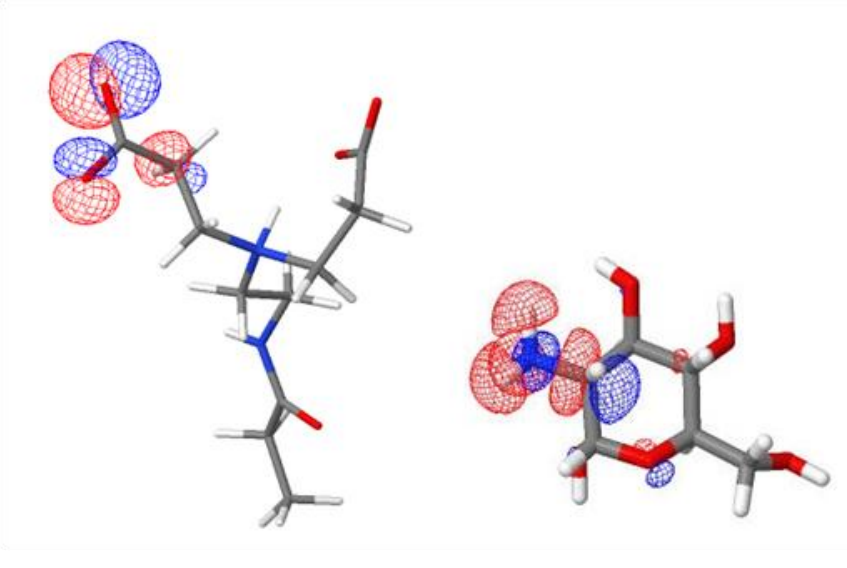




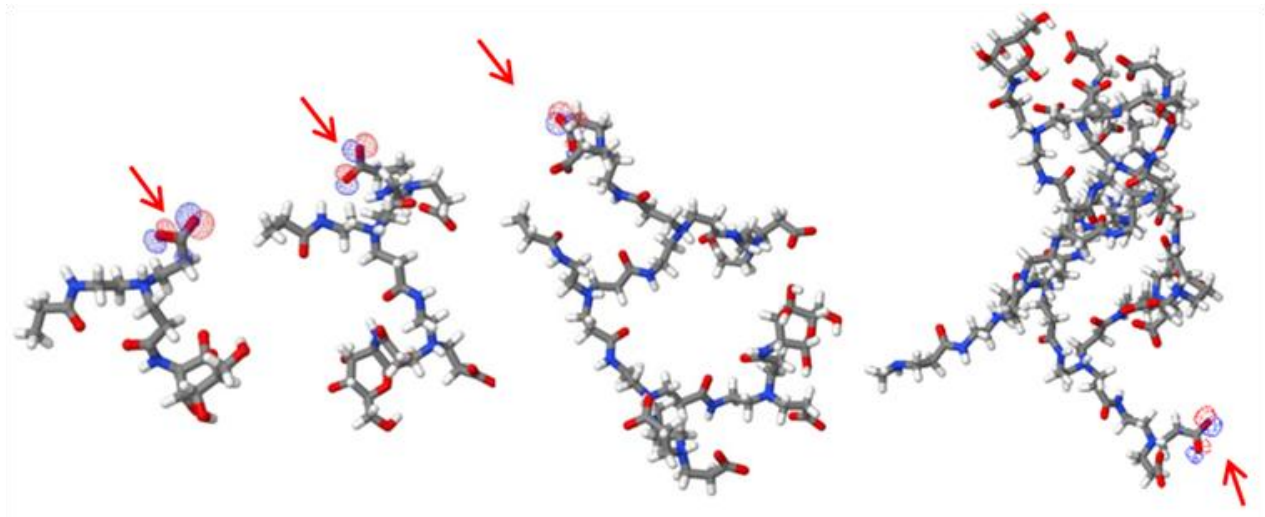

HOMO $^{\mathrm{d}}$-LUMO ${ }^{\mathrm{g}}$ GAP $(\mathrm{eV})$

7.83

5.00

3.13

2.71 


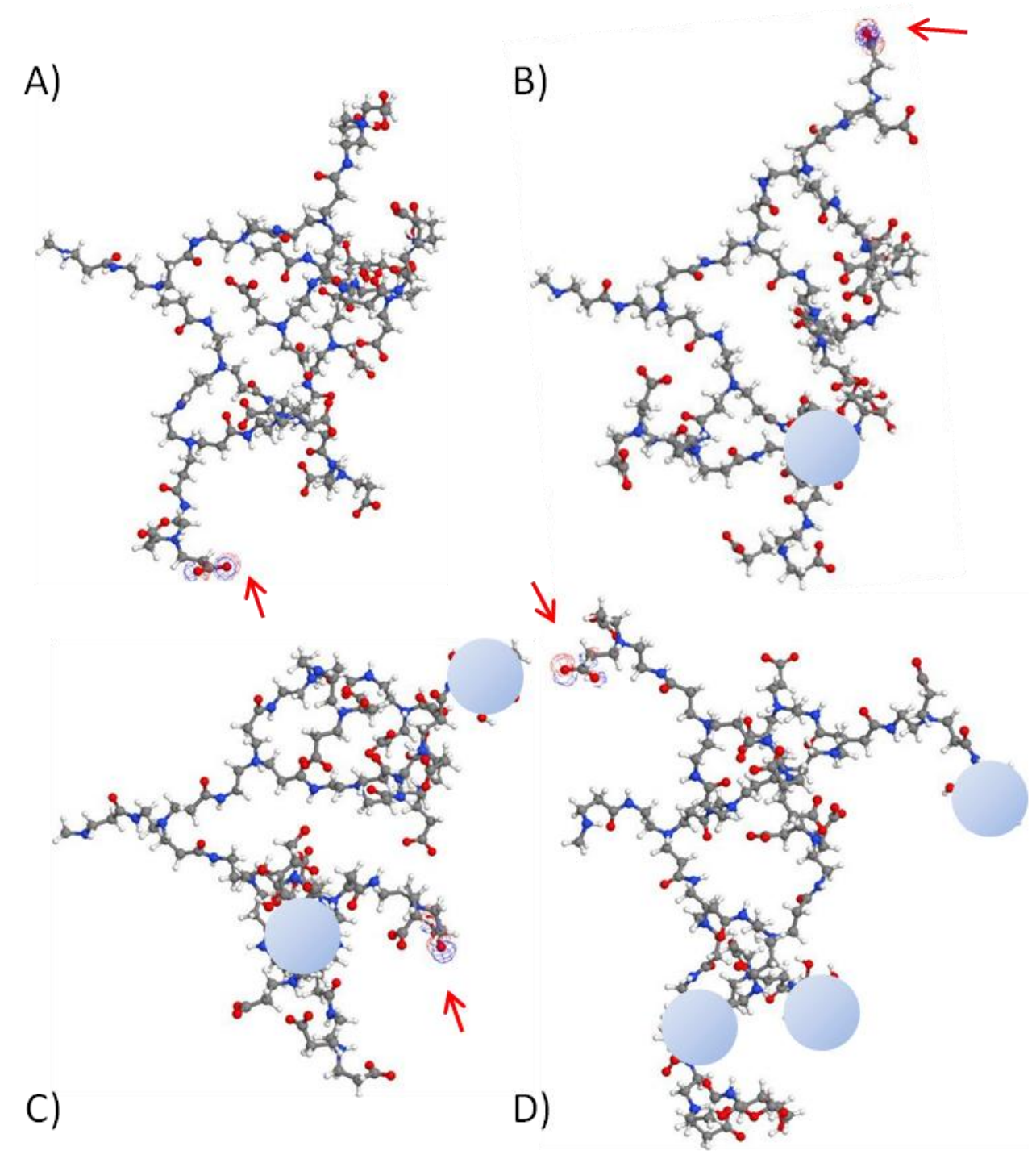



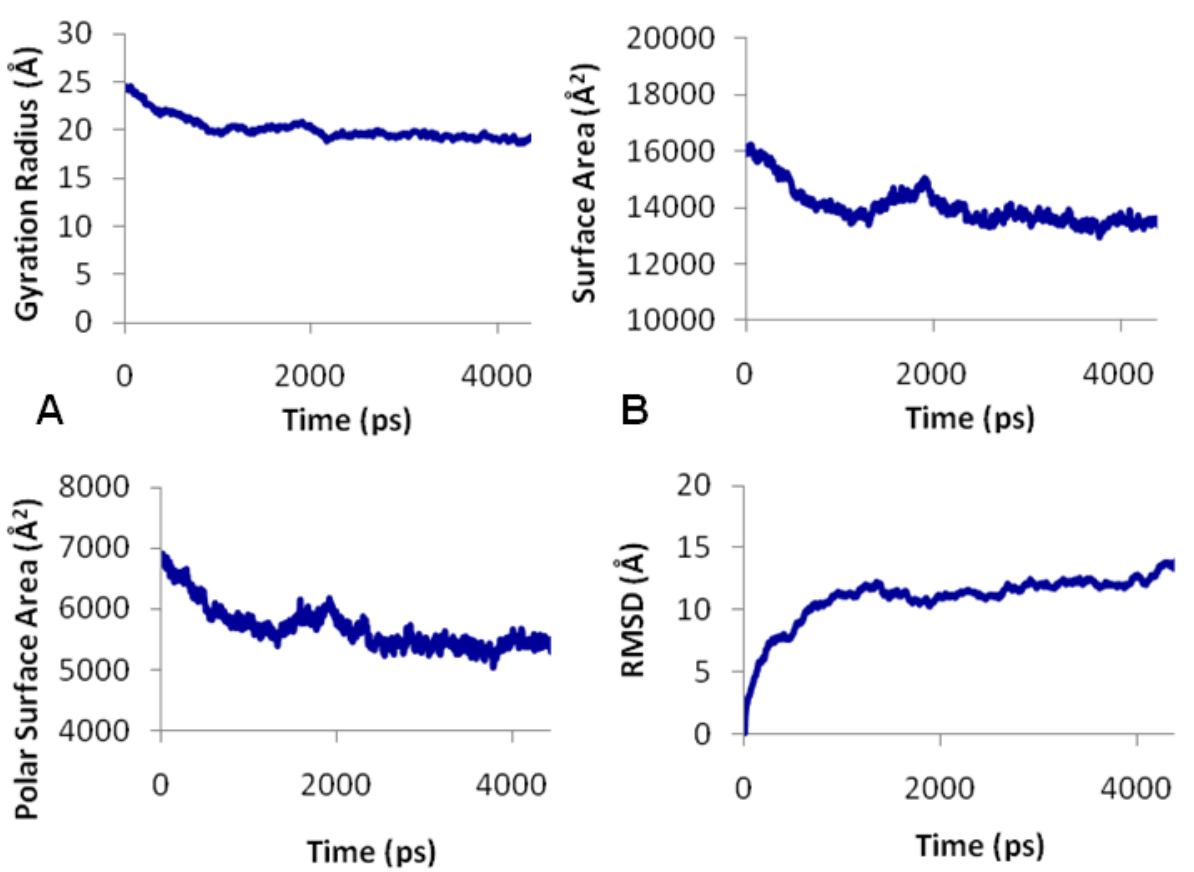

C

D 


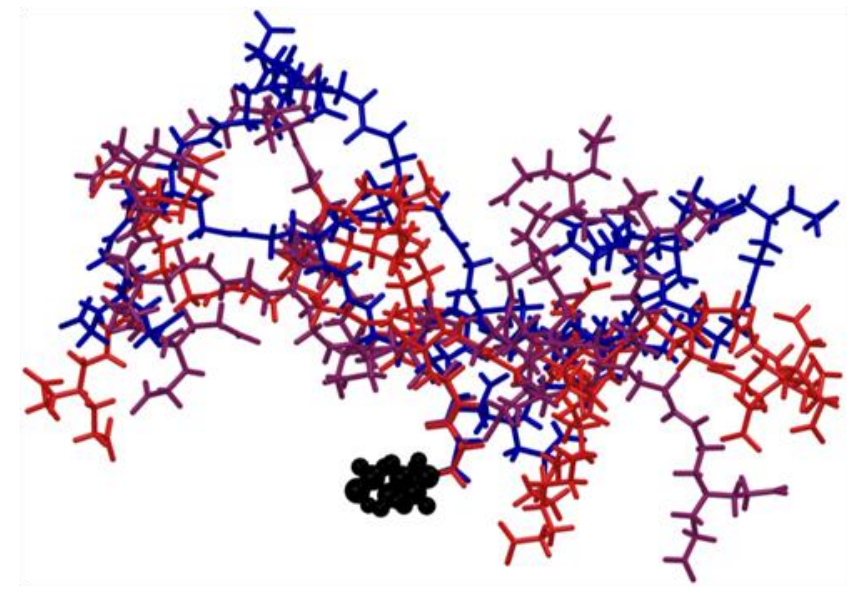



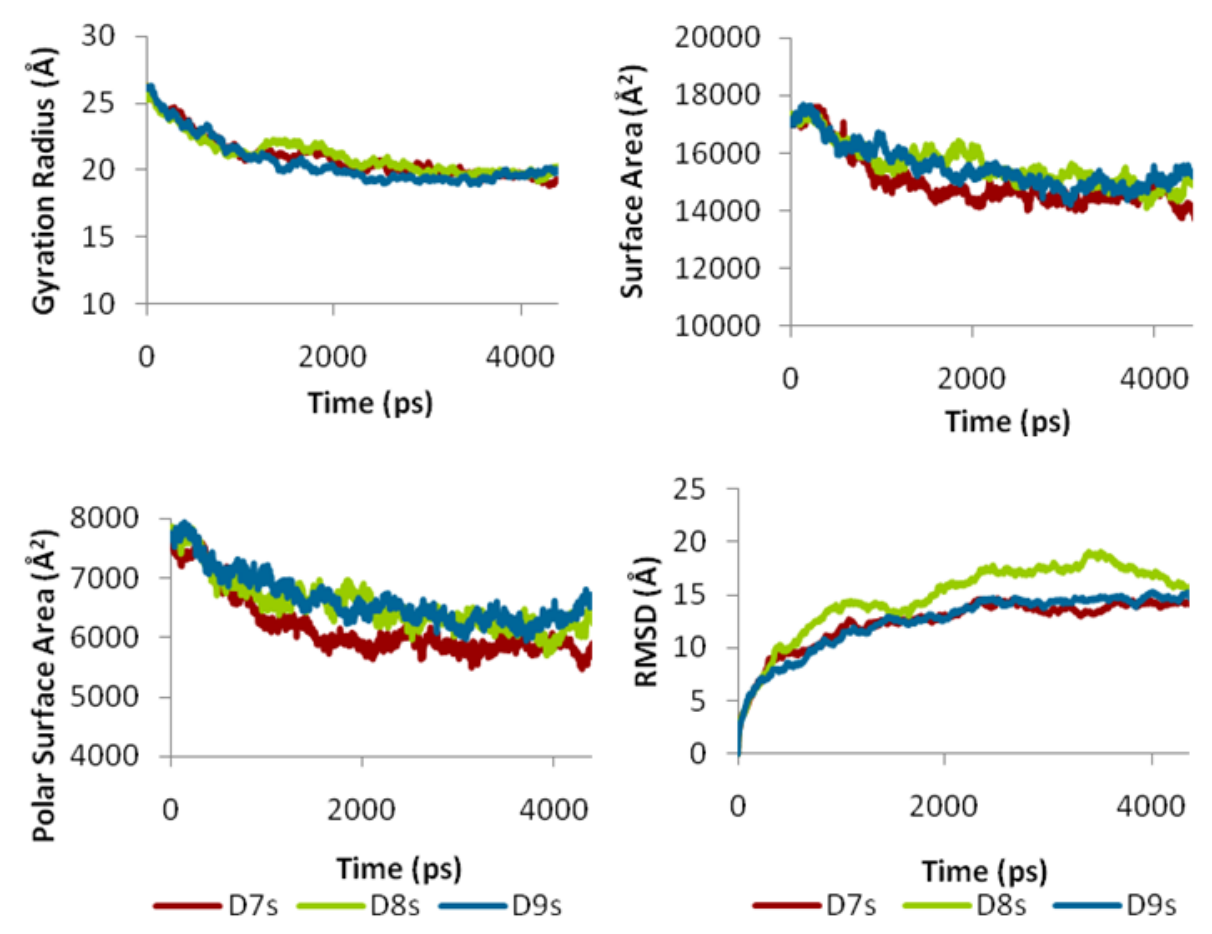


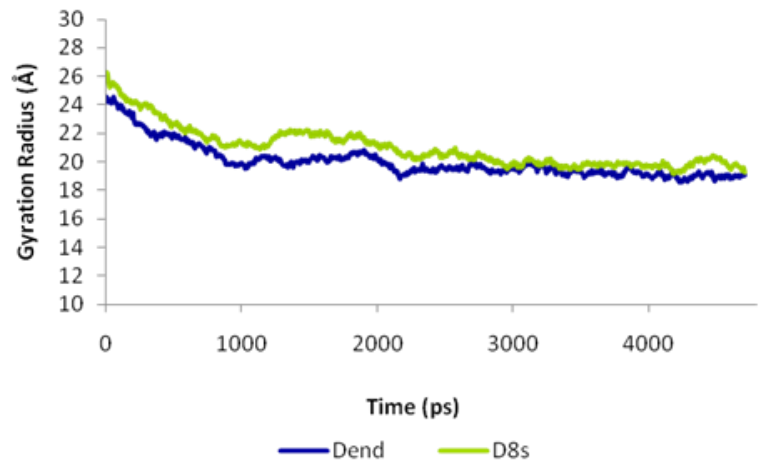


26

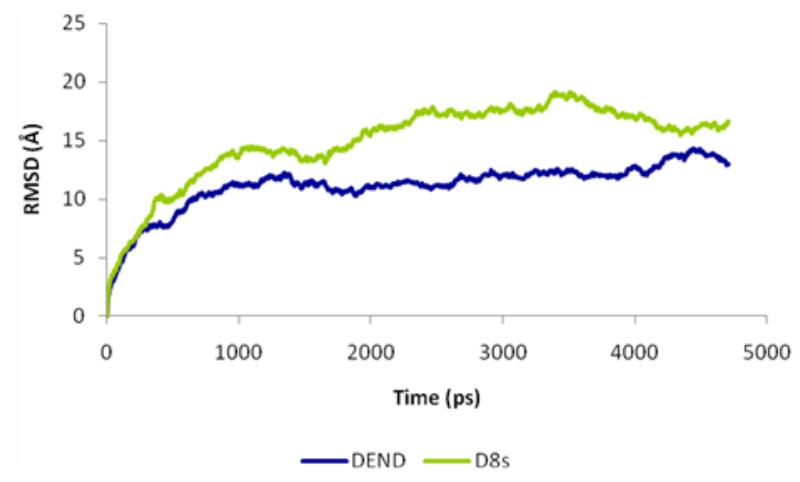




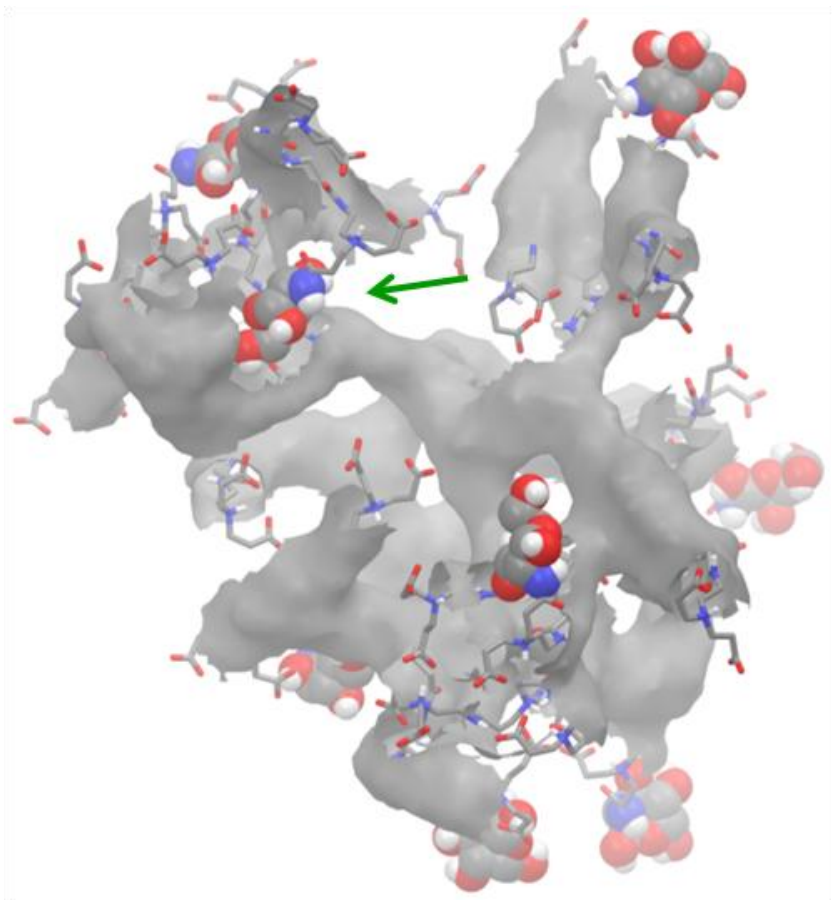




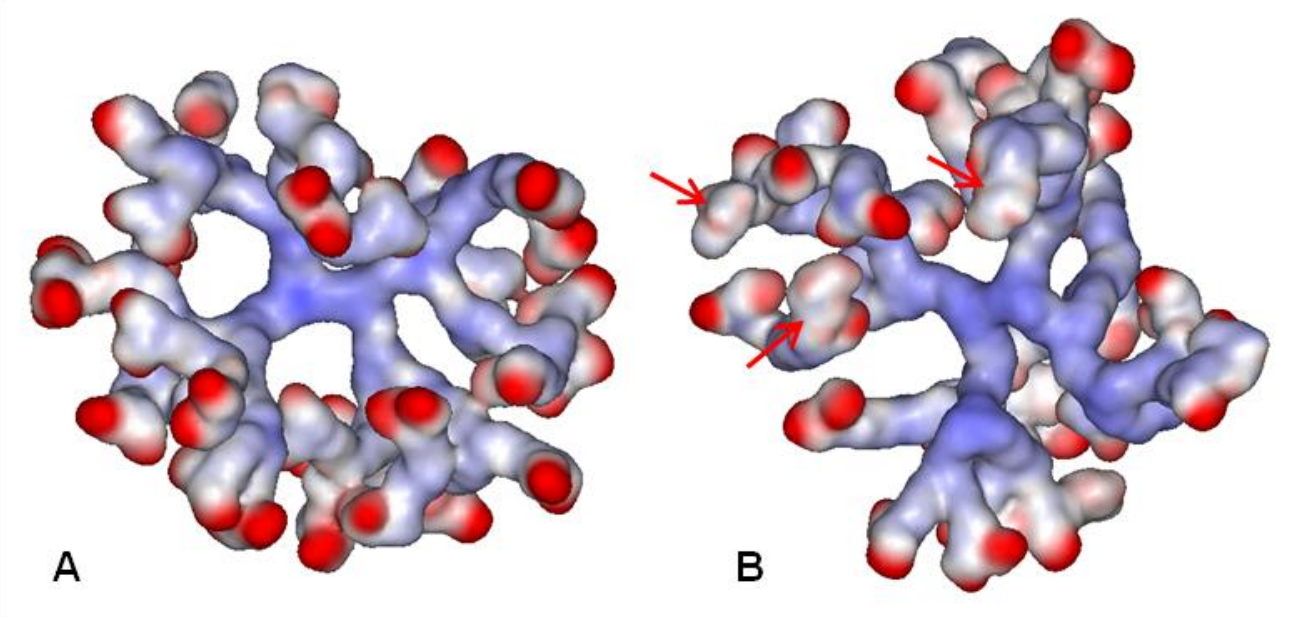


$\mathbb{O}_{\mathrm{N}=\mathrm{C}=\mathrm{N}}^{\mathrm{O}} \longrightarrow$

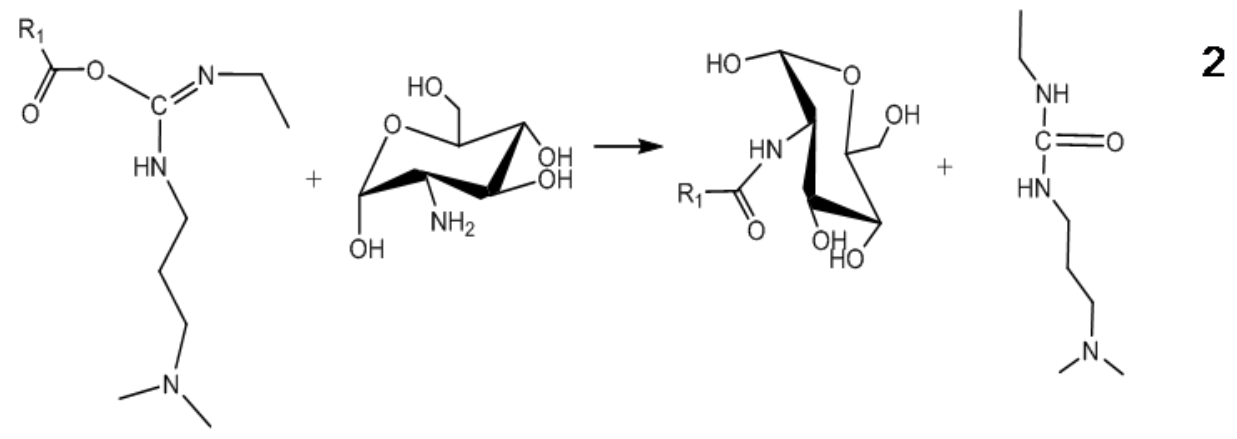

